\title{
Valutazione in parallelo e in serie di test diagnostici multipli
}

\author{
Francesco Franco ${ }^{1}$, Anteo Di Napoli ${ }^{2}$ \\ ${ }^{1}$ Regione Lazio, Roma \\ ${ }^{2}$ Comitato Tecnico-Scientifico RIDT, Roma
}

\begin{abstract}
Evaluation of diagnostic tests in parallel and in series
Multiple diagnostic tests are often required in clinical setting, evaluating the combination of their results to diagnose a disease or a condition. Multiple tests can be performed in parallel or in series. In parallel testing, two or more tests are applied to a patient at the same time. If any of the test results are positive, then the patient is considered to be positive. In series testing, the tests are performed sequentially, but only the individuals that are positive to the initial test are retested. A patient is only considered to be positive if all tests are positive. Using tests in parallel improves the negative predictive value, as it increases the sensitivity and reduces false negatives. Using tests in series improves the positive predictive value, as it increases specificity and reduces false positives.
\end{abstract}

Keywords: Clinical epidemiology, Multiple tests, Tests in parallel, Tests in series

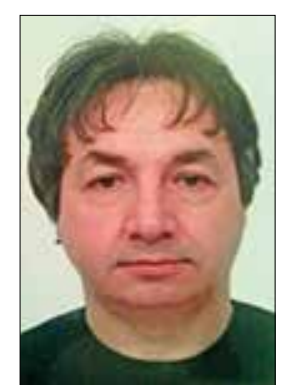

Francesco Franco

\section{Introduzione}

Nei numeri precedenti abbiamo sinteticamente illustrato le caratteristiche di un test diagnostico che concorrono a definirne la validità, vale a dire la capacità di stabilire la presenza o l'assenza di una malattia a seconda della sua positività o negatività, approfondendo il valore predittivo che il medico può attribuire all'esito del test rispetto a un quesito diagnostico, in considerazione della prevalenza della patologia sulla quale si sta indagando (1). Inoltre, abbiamo trattato il tema del rapporto di verosimiglianza di un test diagnostico anche alla luce del teorema di Bayes (2).

Per concludere questa serie di articoli dedicati alla validità di un test diagnostico, tratteremo il tema della valutazione dell'utilizzo di test diagnostici multipli, che rappresenta la si-

Accepted: June 21, 2016

Published online: July 11, 2016

Indirizzo per la corrispondenza:

Dr. Anteo Di Napoli

anteo.dinapoli@libero.it

Dr. Francesco Franco

franco_francesco@fastwebnet.it tuazione che più concretamente si verifica nella realtà clinica.

In generale, l'utilizzo clinico di un test diagnostico in un soggetto sintomatico è finalizzato all'esclusione di alcune ipotesi diagnostiche compatibili con il quadro clinico o alla conferma di un'ipotesi diagnostica fortemente avvalorata dal complesso dei sintomi.

Quando ci si trova di fronte a un quadro clinico compatibile con più ipotesi diagnostiche, è necessaria l'esclusione di alcune di esse, anche sulla base delle risposte negative a un primo test somministrato al paziente; pertanto, poiché ci si deve fidare dei risultati negativi del test, questo deve avere valore predittivo negativo e sensibilità elevati, per ridurre la probabilità di un falso negativo (3).

Quando si opera su pazienti con un quadro clinico suggestivo di una precisa ipotesi diagnostica, è necessaria la sua conferma, anche sulla base delle risposte positive a un primo test; dal momento che ci si deve fidare dei risultati positivi del test, questo deve avere valore predittivo positivo e specificità elevati, per ridurre la probabilità di un falso positivo (3).

Nel caso, invece, di un test finalizzato alla scoperta di una malattia in soggetti asintomatici, come nel caso di uno screening, è necessario un test con elevata sensibilità per individuare la malattia ogni volta che è presente, ma anche di accettabile specificità, al fine di evitare l'aumento dei costi del programma di screening a causa di un alto numero di falsi positivi (3).

Indipendentemente dalle finalità, non è comunque frequente che il medico affidi a un singolo test la risposta al proprio quesito diagnostico.

In condizioni di emergenza, è più comune che si faccia ricor- 
so alla somministrazione "in parallelo" di una batteria di test, al fine di ottenere informazioni diagnostiche tempestive (3-5).

In scenari meno condizionati dall'urgenza, il più delle volte il processo diagnostico si caratterizza per la somministrazione "in serie" di test a pazienti risultati positivi al primo e/o a successivi esami. In molti casi, inoltre, la pratica clinica si caratterizza per un utilizzo combinato delle due strategie diagnostiche, dove, a un iniziale approccio di somministrazione di test "in parallelo", può seguire quello "in serie" (3-5).

\section{Test diagnostici in parallelo}

La somministrazione di test diagnostici in parallelo è tipica delle condizioni di emergenza, per esempio nei Pronto Soccorso, quando è necessario porre diagnosi nel più breve tempo possibile (3-5).

Nel caso della somministrazione di test diagnostici in parallelo, il paziente sarà considerato ammalato se almeno uno di due o più test risulterà positivo (interpretazione dei risultati in chiave "OR"). In tale contesto, la valutazione in parallelo dei risultati di una batteria di test, dal momento che aumenta la probabilità che un paziente risulti positivo al quesito diagnostico, rispetto ai singoli test, determinerà una maggiore sensibilità, per la riduzione dei falsi negativi, e una minore specificità, per l'aumento dei falsi positivi. Infatti, oltre alle situazioni di emergenza, la somministrazione in parallelo è riservata anche ai casi in cui si utilizzino test che abbiano bassa sensibilità (3-5).

Un approccio diagnostico in parallelo in chiave "OR", confidando sulla positività a un singolo test per porre diagnosi di malattia, si caratterizza per un basso valore predittivo positivo, poiché il medico riporrà meno fiducia in un singolo test risultato positivo in presenza, eventualmente, di risultati negativi di altri esami. Al contrario, la somministrazione di test diagnostici in parallelo, in chiave "OR", determina un elevato valore predittivo negativo, poiché il medico potrà escludere la diagnosi con il conforto di test risultati tutti negativi. In questo caso, l'impiego di test multipli in parallelo è particolarmen- te indicato nelle circostanze in cui sia necessario avere una ragionevole certezza di non essere in presenza di un caso di falso negativo (3-5).

Per riassumere, l'applicazione di due o più test in parallelo pone un problema di interpretazione dei risultati ottenuti in chiave alternativa (6).

Per esempio, considerando i risultati di due test, vi sono 4 possibili combinazioni:

a) test-1 positivo \& test-2 negativo

b) test-1 negativo \& test- 2 positivo

c) test-1 positivo \& test-2 positivo

d) test-1 negativo \& test-2 negativo

Le situazioni ai punti c) e d) non creano dubbi nel fare diagnosi (i due test sono concordanti), mentre quelle dei punti a) e b), mostrando una discordanza nei risultati, richiedono l'applicazione di un criterio di valutazione.

In tali situazioni, due sono le possibili chiavi di interpretazione:

1) interpretazione dei risultati in chiave " $O R$ ";

2) interpretazione dei risultati in chiave " $A N D$ ".

Secondo l'interpretazione in chiave "OR", una persona che è risultata essere positiva ad almeno uno dei due test è considerata essere malata. Questa interpretazione aumenta la sensibilità del test congiunto ma ne diminuisce la specificità. Ciò è intuitivo, in quanto una persona ha una probabilità maggiore di risultare positiva.

Secondo l'interpretazione in chiave "AND", una persona è considerata essere malata solo se è risultata positiva a entrambi i test. Questa interpretazione consente di aumentare la specificità del test congiunto, in quanto, per ciascuna persona, la probabilità di risultare positiva a entrambi i test è inferiore rispetto a quanto avviene nell'interpretazione in chiave "OR".

Nel Riquadro 1 è presentato un esempio di calcolo di sen-

RIQUADRO 1 - Test multipli in parallelo (caso di 2 test) combinati in chiave "OR" e "AND"

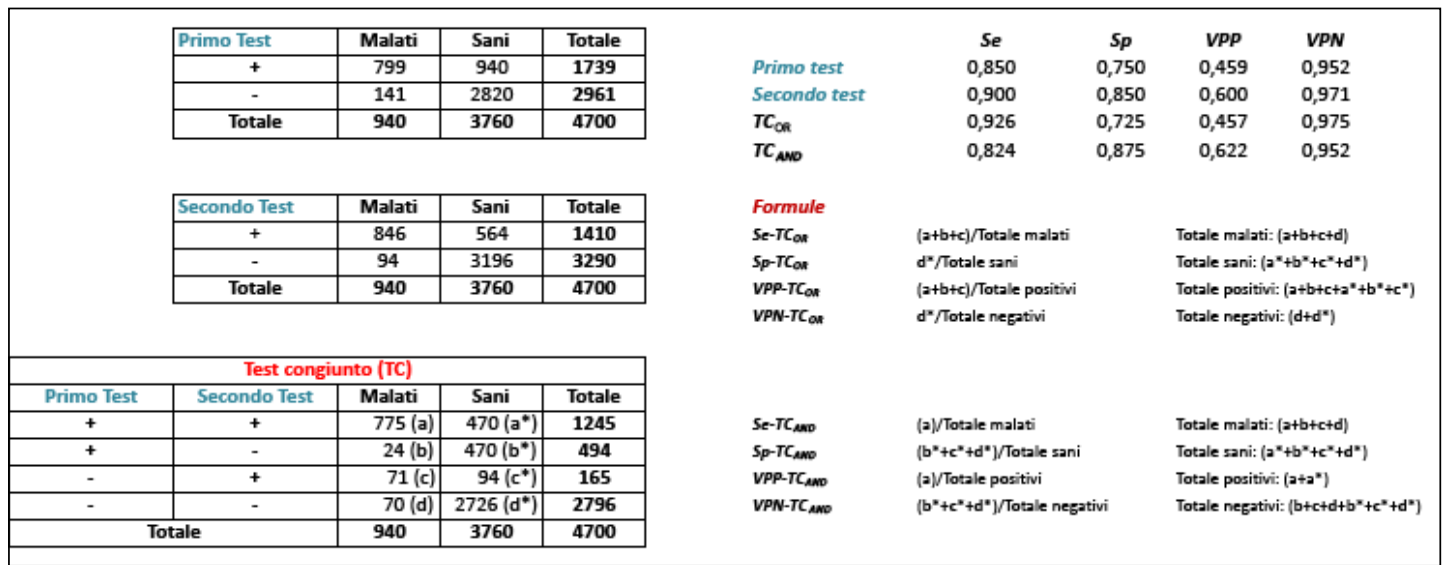


RIQUADRO 2 - Test multipli in serie (caso di 2 test) per i soggetti risultati "negativi" al primo test e per i soggetti risultati "positivi" al primo test

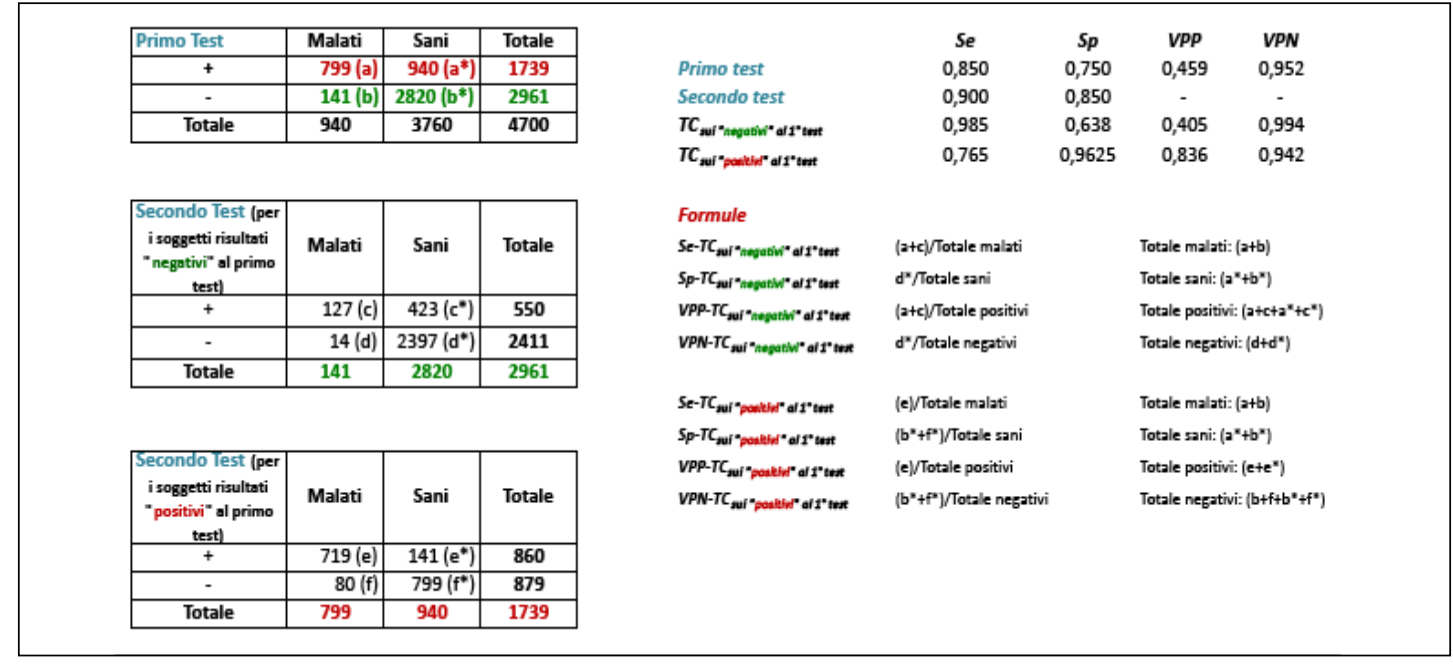

sibilità, specificità e valore predittivo positivo e negativo di test multipli somministrati in parallelo, nell'ipotesi di 2 test, considerando le possibili combinazioni interpretative in chiave "OR" e "AND".

\section{Test diagnostici in serie}

La somministrazione di test diagnostici in serie è tipica delle situazioni in cui sia necessario avviare un paziente in un percorso diagnostico caratterizzato da esami sempre più approfonditi e indaginosi, per esempio nel caso dei programmi di screening. La somministrazione di test diagnostici in serie permette di riservare i test sempre più specifici (spesso costosi e pericolosi) solo a determinati tipi di pazienti, in particolare a quelli risultati positivi a test precedenti e, pertanto, con una più alta probabilità di malattia (3-5).

Infatti, l'esecuzione di test in serie trova applicazione in due distinte situazioni (6):

a) quando dei soggetti devono essere sottoposti a test diagnostici invasivi o molto costosi; in tale circostanza, è opportuno individuare quali soggetti sottoporre al test invasivo e sarà appropriato selezionare solo quelli già risultati positivi a un primo test diagnostico (di più facile attuazione) e che necessitano di ulteriori approfondimenti (secondo test applicato solo ai soggetti risultati positivi al primo test).

b) quando si è in presenza di soggetti particolarmente a rischio per una determinata patologia e che, a un primo test diagnostico, di non elevata sensibilità, siano risultati negativi (secondo test applicato solo ai soggetti risultati negativi al primo test).
Nel caso della somministrazione di test diagnostici in serie, il paziente sarà considerato ammalato se due o più test risulteranno tutti positivi. In tale situazione, il processo diagnostico è riservato solo ai pazienti risultati positivi al primo test e ogni successivo esame sarà eseguito solo se quelli precedenti sono risultati positivi (3-5).

Pertanto, la valutazione in serie dei risultati di successivi test, dal momento che aumenta la probabilità che un paziente risulti negativo al quesito diagnostico, determinerà una maggiore specificità, per la riduzione dei falsi positivi, e una minore sensibilità, per l'aumento di falsi negativi (3-5).

Un approccio diagnostico in serie, applicato solo ai soggetti risultati positivi al primo test (ed eventualmente ai successivi), si caratterizza per un elevato valore predittivo positivo, in considerazione del fatto che la diagnosi di malattia viene posta al termine di un percorso dove test successivi sono risultati tutti positivi. Al contrario, questo tipo di somministrazione di test diagnostici in serie determina un basso valore predittivo negativo, in quanto una diagnosi sarà esclusa sulla base del risultato di un singolo test negativo, successivo ad altri risultati invece positivi (3-5).

L'utilizzo di test in serie sui soggetti risultati positivi al primo test aumenta la specificità complessiva del percorso diagnostico, rispetto a quella dei singoli test, a scapito della sensibilità. Ė, tuttavia, un dazio che deriva dalla necessità di avere la certezza di un risultato positivo, in considerazione del fatto che spesso la conseguenza di un falso positivo è l'invio del soggetto a un intervento chirurgico e che, nel caso dei programmi di screening, i test diagnostici sono rivolti a persone asintomatiche, in apparente buona salute. Inoltre, considerando che un programma di screening prevede controlli periodici, gli eventuali falsi negativi avranno ulteriori possibilità di essere individuati e corret- 
tamente diagnosticati (3-5).

Nel Riquadro 2 è presentato un esempio di calcolo di sensibilità, specificità e valore predittivo positivo e negativo di test multipli somministrati in serie, nell'ipotesi di 2 test, per i soggetti risultati "negativi" al primo test e per i soggetti risultati "positivi" al primo test.

\section{Disclosures}

Financial support: No financial support was received for this submission.

Conflict of interest: The authors have no conflict of interest.

\section{Bibliografia}

1. Franco F, Di Napoli A. Introduzione alla valutazione di un test diagnostico: sensibilità, specificità, valore predittivo. Giornale di Tecniche Nefrologiche e Dialitiche. 2016;28(1):53-5.

2. Franco F, Di Napoli A. Rapporto di verosimiglianza del risultato positivo e negativo di un test diagnostico e teorema di Bayes. Giornale di Tecniche Nefrologiche e Dialitiche. 2016;28(2):134-6.

3. Carle F, Mattei A, Necozione F, Di lorio F. Caratteristiche del test diagnostico. In: Di lorio F, a cura di, Elementi di metodologia epidemiologica clinica. Padova: Piccin Editore. 1994:60-76.

4. De Masi S. Validità e riproducibilità delle misure: la diagnosi. In: Mele A, Spada E, Tosti ME, a cura di, Epidemiologia per la clinica e la sanità pubblica. Roma: II Pensiero Scientifico Editore. 2009: 27-41.

5. Cebul RD, Hershey JC, Williams SV. Using multiple tests: series and parallel approaches. Clin Lab Med. 1982;2(4):871-90.

6. Cesana BM, Antonelli P, Pea G. La Statistica per le Scienze Biomediche. Padova: libreriauniversitaria.it Edizioni 2012; Tomo I, Capitolo. 6:423-42. 